\title{
Powdering Up for Neutrinos
}

\author{
The search for neutrinos from past supernovae is getting an upgrade as \\ Japan's Super-Kamiokande experiment begins adding gadolinium \\ powder to its giant water-based detector.
}

By Michael Schirber

S upernovae are some of the brightest objects in the Universe, not only in light but also in neutrinos. In 1987, neutrino experiments got their first sampling of these supernova neutrinos from a stellar explosion in a nearby galaxy. Since then scientists have waited-in vain-for the next nearby blast. However, the sky is currently full of "fossil" neutrinos from all the supernovae that went off in the past. The Super-Kamiokande experiment in Japan has been hunting for these relic neutrinos but so far hasn't had any luck. To boost their sensitivity, the experiment has started mixing gadolinium powder into their 50,000-ton water detector. If all goes according to plan, this upgrade should yield the first signs of relic neutrinos in the next few years.

Core-collapse supernovae occur when a massive star runs out of fuel and falls in on itself because of gravity. The resulting

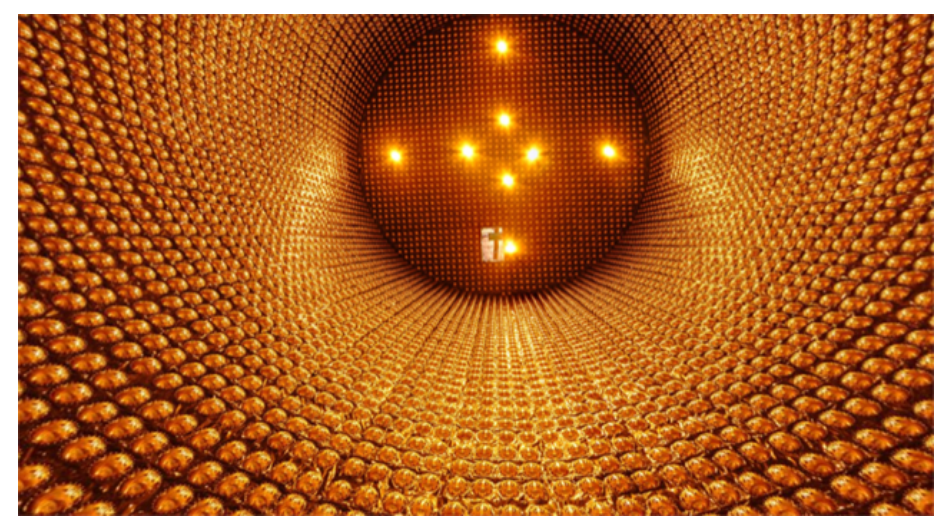

A view from the bottom of the Super-Kamiokande detector during refurbishment in 2018.

Credit: Kamioka Observatory, ICRR, the University of Tokyo/NHK Enterprises, INC.

explosion sets off a burst of light that rivals an entire galaxy of stars. But most of the fireworks happen in particles that we can't see. "Almost all of the gravitational-collapse energy comes out in neutrinos," says John Beacom from the Ohio State University. Astronomers are very interested in studying these escaping neutrinos, as they could provide missing clues about the mechanism that drives the explosion. "We can't understand core-collapse supernovae until we detect their neutrinos," Beacom says.

Despite the large number of neutrinos emitted from a supernova, they are almost impossible to detect except for relatively close events. The 1987 event was a core-collapse supernova that occurred in a dwarf galaxy located just on the outskirts of the Milky Way and was observed by multiple neutrino detectors as a spike of 24 neutrinos. From that one event, scientists were able to estimate the total energy carried away from the supernova by neutrinos. But they'd like to observe more of these events to have a better sense of the typical energy distribution-or spectrum-of supernova neutrinos. Unfortunately, our local cosmic neighborhood is not well stocked with massive, supernova-prone stars. "You know, I've been holding my breath since 1987," Beacom says. "And it's just not working out all that good."

But Beacom says that there is a way to potentially see supernova neutrinos sooner by targeting the relic neutrinos from all the past supernovae in cosmic history. This "background" fills the Universe with an energy density that is comparable to the energy density of light from all the stars that ever existed. In terms of the flux, models estimate that a few relic neutrinos per centimeter squared per second should strike Earth's surface. The only detector capable of detecting that flux is Super-Kamiokande, Beacom says. 
Over the past two decades, Super-Kamiokande, or Super-K for short, has searched for events in which a relic neutrino collides with one of the protons in the facility's 41-m-tall water tank, producing a positron and a neutron. The neutron flits around in the water and eventually connects with a hydrogen nucleus, producing a hard-to-detect gamma ray. By contrast, the fast-moving positron generates Čerenkov light that can be measured by the 13,000 photodetectors lining the tank's walls. The challenge is that similar Čerenkov signals are produced by cosmic rays and "local" neutrinos from the Sun, atmosphere, and nuclear reactors. Only about 1 out of every 10,000 signals is a supernova relic neutrino.

"It is like looking for a needle in a haystack," says Sonia El Hedri from the École Polytechnique in France. She and her colleagues developed a strategy to tag neutron-producing events, which would effectively single out supernova neutrinos. A month ago at the (virtual) Neutrino 2020 conference, El Hedri presented their results-no detections, but the sensitivity to supernova relics is closing in within a factor of 2 of theoretical predictions.

To improve this sensitivity, the Super-K Collaboration is embarking on a radical change to their detector. The plan-originally described in a 2004 paper by Beacom and Mark Vagins from the University of California, Irvine-is to add gadolinium to Super-K's otherwise ultrapure water. The gadolinium nucleus captures neutrons much more readily than the hydrogen nucleus. "It is freakishly hungry for neutrons," Vagins says. And when a neutron is captured by gadolinium, it makes an energetic gamma-ray signal that is easy to spot.

On July 14, Super-K started dissolving 13 tons of gadolinium sulfate octahydrate in water and injecting it at the bottom of the tank. "We've been watching the 'gadolinium front' slowly move up through the water," says Vagins, who is in Japan with a joint appointment at the University of Tokyo. He expects the loading will be done by the end of August, at which point the gadolinium concentration will be $0.01 \%$ by mass.

Expectations are that gadolinium-enriched Super-K will detect around three supernova relic neutrinos per year. Vagins thinks that within four to six years they will have enough data to report a scientifically interesting result. He says the great thing about supernova relic neutrinos is that they represent an average over all the explosions of the past. "So, we're absolutely going to learn what the typical supernova looks like."

Michael Schirber is a Corresponding Editor for Physics based in Lyon, France. 